\title{
Potentials and Limits of Plasticity Induced by Working Memory Training in Old-Old Age
}

\author{
Katharina Zinke $^{\mathrm{a}}$ Melanie Zeintl $^{\mathrm{a}} \quad$ Anne Eschen $^{\mathrm{b}}$ Carole Herzog ${ }^{\mathrm{c}}$ \\ Matthias Kliegel $^{\mathrm{a}}$

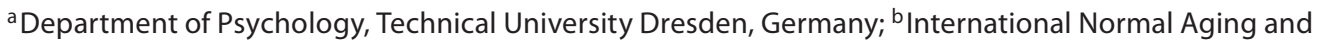 \\ Plasticity Imaging Center (INAPIC) and ' Department of Psychology, University of Zurich, Switzerland
}

\section{Key Words}

Memory $\cdot$ Executive functions $\cdot$ Plasticity $\cdot$ Fourth age

\begin{abstract}
Background: Old-old age (80+ years) is associated with substantial cognitive decline. In this population, training-induced cognitive plasticity has rarely been studied. While earlier findings on strategy trainings suggested reduced training gains in old-old age, recent results of an extensive process-based working memory (WM) training have been more positive. Objective: Following up on previous research, the present study aimed at examining the effects of a short WM training in old-old adults and the influence of baseline WM capacity on training gains. Methods: A training group (mean age: 86.8 years) and a matched control group (mean age: 87.1 years) participated in the study. The WM training consisted of five tasks that were trained in each of 10 sessions. To evaluate possible transfer effects, executive functions were assessed with two tests before and after training. The training group was divided via median split in high- and low-capacity individuals to determine the influence of baseline WM capacity on training gains. Results: The training group improved in four of the trained tasks (medium-to-
\end{abstract}

large effects). Training gains were significantly larger in the training group than in the control group in only two of those tasks. The training effects were mainly driven by the lowcapacity individuals who improved in all trained tasks. No transfer effects were observed. Conclusions: These positive effects of a short WM training, particularly for low-capacity individuals, emphasize the potential for cognitive plasticity in old-old age. The absence of transfer effects may also point to its limits.

Copyright $\odot 2011$ S. Karger AG, Basel

\section{Introduction}

Aging is associated with functional loss in many cognitive domains, in particular processing speed, memory, and executive functions [1]. For a long time, the possible modifiability of this functional decline in old age, in particular through cognitive training interventions, has received a lot of interest in gerontology and geriatrics. This line of research has led to mainly positive news; especially for young-old adults (60-80 years). Young-old adults seem to be able to recruit effective encoding and retrieval strategies to compensate for some aspects of the cogni-

\section{KARGER \\ Fax +4161306 1234 E-Mail karger@karger.ch} www.karger.com

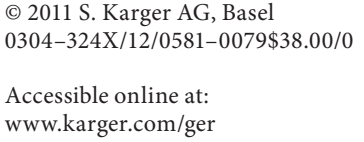

Katharina Zinke

Department of Psychology

Technical University Dresden

DE-01062 Dresden (Germany)

Tel. +49 351463 39193, E-Mail zinke@psychologie.tu-dresden.de 
tive losses arising during late life: a range of training studies suggest that in young-old adults episodic memory capacity can be enhanced through teaching mnemonic strategies (e.g. the method of loci [2]). In contrast, although there are far fewer studies targeting old-old adults (80+ years), this age has been described as an age of substantial loss in cognitive plasticity. Available reports clearly suggest a decline of old-old adults' ability to benefit from strategy-based interventions, with considerably smaller improvements obtained in those interventions for old-old adults as compared to young-old adults [3].

Over the last few years, a promising conceptual alternative to strategy-based episodic memory training interventions has emerged which focuses on training working memory (WM) capacity. WM has been defined as the ability to maintain (store) and manipulate (process) information within short periods of time and comprises a verbal (phonological), a visuospatial, and an executive subsystem [4]. WM is a central neurocognitive processing resource that is involved in most conscious everyday mental activities. It is thought to support a wide range of complex cognitive activities, including logical reasoning and problem solving, has been shown to be strongly related to measures of fluid intelligence $[5,6]$, and is even involved in emotion regulation and social interactions $[4,6]$.

Given its importance for cognitive functioning, it is rather surprising that so far research on WM training is scarce [4-6]. One reason for the scarcity of WM training studies may be that, until recently, WM capacity had been conceptualized as a stable trait representing interindividual differences [6]. However, the rationale for newer process-oriented training studies is that WM performance may be improved through repeated practice on WM tasks (rather than through teaching strategies). Secondly, as $\mathrm{WM}$ is involved in a wide range of cognitive functions, training gains in WM may possibly lead to improvements in those functions also. Indeed, recent training studies targeting WM processes through repeated practice have revealed promising results in this regard. They have been able to show improvements in WM functions for children with ADHD, children with low WM skills $[7,8]$ and young adults [9]. Additionally, some studies have also provided preliminary evidence for transfer effects to nontrained tasks [7, 9].

Importantly for present purposes, very recently those effects have also been shown for older adults. Besides its general importance for human cognitive functioning, the possibility of improving WM capacity in older adults is of particular relevance for gerontology and geriatrics as cognitive aging research has revealed substantial mean level decreases in WM in old age (in both verbal and spatial WM tasks) $[1,10]$. Particularly in old-old adults, WM capacity has been shown to decrease substantially $[11,12]$. However, until now only a handful of WM training studies have been conducted with participants of an older age range, and only one of them has approached the old-old age range. Those studies provided evidence for some training-induced plasticity in young-old adults $(60-80$ years), for example by applying an executive control training using a task switching paradigm [13]. Also, after 5 weeks of training on an updating task (i.e. recalling the last items out of sequences of consecutively presented items), young-old adults with a mean age of 68 were comparable in their performance gains on the trained task to a young control group [14]. Similarly, Li et al. [15] found improved performance in young-old adults with a mean age of 75 after 6 weeks of training on a visuospatial n-back task. So far, only one study has investigated plasticity of WM on the edge to old-old age, with promising results: after a 3-month intervention to train visuospatial WM, a group of 80 -year-old adults improved their WM performance on all trained tasks [16].

Findings on transfer effects in all the mentioned studies, however, were heterogeneous and suggest that transfer may be restricted. Dahlin et al. [14] found transfer after their updating training only in their young participants. Buschkuehl et al. [16] found a clear transfer effect only for a visuospatial WM task, not for a verbal one. Since their WM training tasks were all visuospatial, their transfer effects seem to be specific to the type of memory content. Although Li et al. [15] demonstrated transfer of their visuospatial n-back WM training to visuospatial as well as numerical n-back tasks in both young and old participants, they found no transfer to more complex WM span tasks. Thus, transfer was restricted to WM tasks that were very similar to training tasks with regard to involved processes.

Following up on this recent development, the present study set out to examine a range of open issues that are currently under debate. (1) Because of the small number of WM training studies in old age, it is not yet clear whether plasticity in WM is still preserved in old-old age and whether and to what extent transfer effects may be observed (see Mayr [17] who pointed out the importance of this open issue). The current study focused on far transfer tasks because, although transfer to some similar WM tasks has already been demonstrated, it is not yet clear whether WM training can transfer to more complex (executive control) tasks. (2) Another open question is the 
issue of optimal training time. Most former training studies have used extended periods of training over several weeks or months to achieve effects (e.g. Li et al. [15] trained for 6 weeks; Buschkuehl et al. [16] for 12 weeks). However, both from a theoretical perspective studying underlying processes and from an applied perspective in clinical contexts, in which clinicians are often confronted with time constraints, it is important to explore when those effects emerge and whether shorter durations of training are powerful enough to induce similar effects. Therefore, the second aim of the present study was to explore whether and to what extent adults who are well within the old-old age range can still profit from a WM training that is set up as a considerably shorter training regime. (3) A third research question that the present study aimed to address is whether differential training effects can be observed depending on the participants' functional status before training. In other words: can subgroups of participants be identified who profit more from training than others? In particular, the role of WM ability level at the beginning of training was explored. Considering the previous literature two possible predictions can be derived. On the one hand, it may be that especially in old-old adulthood only those participants who have maintained a certain cognitive status profit from a WM intervention that requires a considerable amount of attentional resources $[18,19,20]$. On the other hand, resting on the disuse hypothesis [21,22], it may be that participants who start with a low cognitive status (a possible result of a decline in using cognitive resources) are able to reactivate some of their potential with the help of training.

\section{Methods}

\section{Participants}

Participants of the study were old-old adults between the ages of 77 and 96. The training group consisted of 20 old-old adults and was matched for age, gender, education, and functional health to a control-group comprising 16 old-old adults (see table 1 for demographic characteristics and cognitive functioning of the two groups). Exclusion criteria were neurological or psychiatric disorders known to cause cognitive dysfunction, in particular mild cognitive impairment (MCI) or Alzheimer's disease (AD). This was screened for with the Mini Mental Status Test (MMST) German short form for old-old adults by Kliegel et al. [23] which takes possible age-associated impairments in visual or motor functioning in extreme old age ranges into account. Maximum sum score on this test is 21 . All individuals scored above the levels indicating cognitive decline [24]. The two groups were comparable in cognitive functioning (MMST) and crystallized intelligence, assessed with a German vocabulary test (MWT) [25].
Table 1. Participant characteristics of the training group $(n=20)$ and the control group $(\mathrm{n}=16)$

\begin{tabular}{|c|c|c|}
\hline & $\begin{array}{l}\text { Training } \\
\text { group }\end{array}$ & $\begin{array}{l}\text { Control } \\
\text { group }\end{array}$ \\
\hline Gender & 14 women & 11 women \\
\hline Age, years & $86.8 \pm 4.9$ & $87.1 \pm 3.7$ \\
\hline Years of education & $11.7 \pm 3.3$ & $10.1 \pm 1.9$ \\
\hline Cognitive functioning $\left(\mathrm{MMST}^{\mathrm{a}}\right)$ & $19.4 \pm 1.4$ & $19.3 \pm 1.5$ \\
\hline Crystallized Intelligence (MWT-A ${ }^{\mathrm{b}}$ ) & $31.1 \pm 3.3$ & $29.4 \pm 3.0$ \\
\hline
\end{tabular}

Mean values \pm SD

${ }^{a}$ MMST = Mini Mental Status Test short form for old-old adults by Kliegel et al. [23] with a maximum score of 21 .

b MWT-A = German Mehrfachwahl-Wortschatz-Intelligenztest version A by Lehrl et al. [25].

\section{Training Tasks}

Following the WM training regime by Klingberg et al. [7, 26], the training material comprised both verbal (Digit span forward and backwards, HAWIE-R [27]) and visuospatial WM tasks (Corsi block tapping forward and backwards [28]; K-ABC Icons, Kaufmann Assessment Battery for Children [29]). Thus, training included tasks that mostly require storage of information (Digit span and Corsi block tapping forward; K-ABC Icons) as well as tasks that additionally require processing components of WM (Digit span and Corsi block tapping backwards). All tasks were administered in individual face-to-face sessions using paper and pencil material instead of a computerized format since the old-old population usually has no computer experience. Difficulty levels were individually adapted because adaptivity has been shown to be an essential feature of effective training regimes [7,26].

In the Digit span task, participants had to repeat numbers that were verbally presented in sequences of increasing length in either the same order (forward) or, in the second half of the task, in reversed order (backwards). In the Corsi block tapping task the experimenter tapped a number of blocks on a board in sequences of increasing length. The participant had to reproduce the sequences by tapping immediately after the experimenter had finished, either in the same order (forward) or, in the second half, in reversed order (backwards). In both tasks, the number of items presented was increased when the participant was successful in completing a sequence. For motivational purposes, the training block ended if the participant failed to recall two sequences of the same number of items correctly. The (modified) K-ABC Icons task is an adaptive visuospatial WM task where the participants had to process and maintain the spatial arrangement of multiple stimuli. An increasing number of pictures of objects (icons) was presented to the participant, first in a $3 \times 3$ grid and then in a $3 \times 4$ grid to adaptively increase WM load. The participant had to memorize the icons and their individual locations within the presentation time of 3 seconds and reproduce the icons and their positions on an empty grid afterwards. The task ended when the participant unsuccessfully completed two items with the same number of icons. 


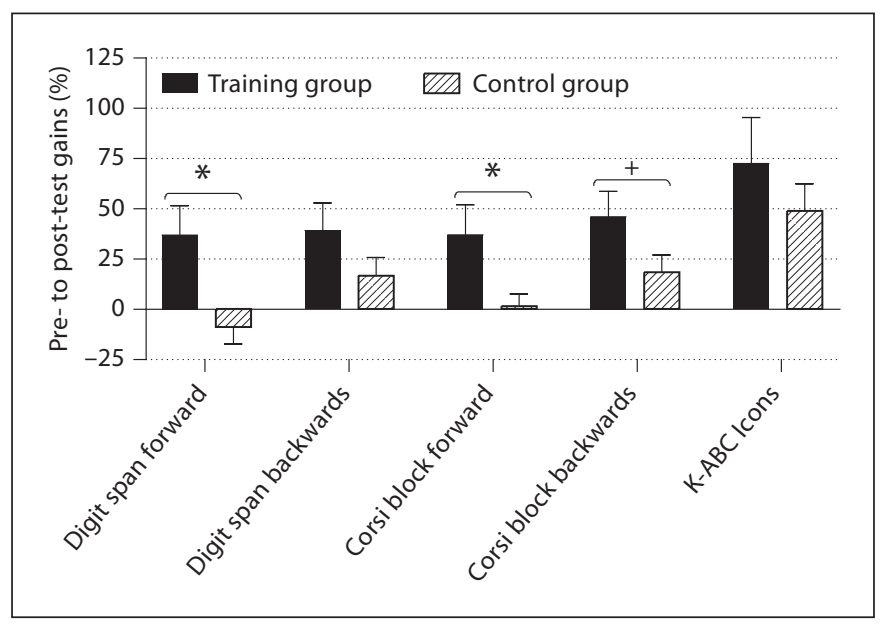

Fig. 1. Comparisons between the training group $(n=20)$ and control group $(n=16)$ for mean training gains in percent for each training task separately. Error bars indicate standard errors. Significant differences between groups are indicated: ${ }^{*} \mathrm{p}<0.05$, $+\mathrm{p}<0.10$.

\section{Training Procedure}

Training was administered in daily sessions of 25-30 min over 2 weeks. To avoid sequence effects, the order of tasks was counterbalanced and varied daily. Parallel versions of the tasks were used each day. The training group received 10 training sessions over 2 weeks, including a weekend break. The compliance rate was high: $96 \%$ of all training task blocks were fully completed. The control group received no treatment.

\section{Assessment of Transfer}

Two to three days before and after training, respectively, the training group completed a pretraining assessment and a posttraining assessment including two standard cognitive tasks to assess far transfer (executive control). Inhibitory control was assessed using a Stroop interference task (German version of the color-word-Stroop test: FWIT [30]). Nonverbal complex reasoning ability was assessed with the Raven Colored Progressive Matrices [31]. These tasks were chosen because they have been shown to be related to $\mathrm{WM}$, the targeted function in the current training regime [5]. The control group was also assessed twice with these tasks, with approximately 2 weeks between the two assessments.

\section{Data Analyses}

Following the studies on WM training by Klingberg et al. [7, 26], paired tests were run for each group to test for significant performance differences between the pre-training and posttraining assessments in all training and transfer tasks. Effect sizes were calculated as Cohen's d, that is, the standardized mean difference in performance between pretest and posttest (pre- to post-test difference divided by the pooled standard deviation). All d values were corrected for small sample bias following Hedges and Olkin ( $\left.\mathrm{d}^{\prime}\right)$ [32]. Then individual performance gains on each training and transfer task (in \%) were compared between groups using independent $t$ tests. To further analyze the course of performance changes during training in the training group a repeated-measures ANOVA with all training days was conducted. Wherever necessary, corrections for the lack of sphericity were applied. Additionally, to further analyze the longitudinal trends observed in the ANOVAs, paired t tests were used to test for differences between performance in the 1st and 5th training sessions (beginning and end of the first training week) and between performance in the 6th and 10th training sessions (beginning and end of second training week). To explore differential training effects, for each training task the training group was divided via median split on pre-training performance into subgroups of high- and low-capacity individuals. Paired t tests were used to analyze performance gains in trained tasks from pre- to post-training in the group of participants with low WM capacity and in the group of participants with high WM capacity separately. To compare the magnitude of training gains between the low and high WM capacity groups and between the low WM capacity training group and the control group independent $t$ tests were applied.

\section{Results}

\section{Comparing Performance in Trained Tasks}

For the training group, paired t tests comparing preto post-WM performance revealed significant performance gains for Digit span forward $(\mathrm{t}(19)=-2.1, \mathrm{p}<0.05$, $\left.\mathrm{d}^{\prime}=0.49\right)$, for Digit span backwards $(\mathrm{t}(19)=-3.0, \mathrm{p}<0.01$, $\left.\mathrm{d}^{\prime}=0.56\right)$, for Corsi block tapping backwards $(\mathrm{t}(19)=-3.8$, $\left.\mathrm{p}<0.001, \mathrm{~d}^{\prime}=0.93\right)$, and for K-ABC Icons $(\mathrm{t}(19)=-4.0$, $\left.\mathrm{p}<0.001, \mathrm{~d}^{\prime}=0.98\right)$. There was a trend towards significance for performance gains in Corsi block tapping forward $\left(\mathrm{t}(19)=-1.8, \mathrm{p}<0.09, \mathrm{~d}^{\prime}=0.47\right)$. According to Cohen's convention [33], effects in Corsi block tapping backwards and $\mathrm{K}-\mathrm{ABC}$ Icons were large, all others were medium. ${ }^{1}$ In contrast, there was only one significant retest effect (K-ABC Icons: $\mathrm{t}(19)=-3.6, \mathrm{p}<0.01, \mathrm{~d}^{\prime}=1.04$ ) for the control group (all other $\mathrm{p}>0.1$ ). Importantly, in comparison to the control group, the training group had significantly higher pre- to post-training gains in percent for Digit span forward (37 vs. $-9 \%, \mathrm{t}(29.0)=2.7, \mathrm{p}<0.01$ ), Corsi block tapping forward (37 vs. 1\%, t(25.3) = 2.2, p < 0.04 ) and there was a trend towards significance for higher pre- to post-training gains in Corsi block tapping test backwards (46 vs. $18 \%, \mathrm{t}(34)=1.7, \mathrm{p}<0.09$; see fig. 1 for descriptive results).

\footnotetext{
1 Preliminary data from a subset of participants in the treatment group $(n=9)$ suggest that training gains remained relatively stable over a period of two weeks: performance in the Corsi block and Digit span tasks forward and backwards, respectively, did not differ significantly between post-test and follow-up 2 weeks later (all $\mathrm{p}>0.05$ ).
} 
In a more detailed analysis of the training progress over the ten training sessions, repeated measures ANOVAs with all ten training sessions were computed. Performance on four of the trained tasks changed significantly across training sessions: for K-ABC Icons, $\mathrm{F}(5.4,75.3)=$ $5.9, \mathrm{p}<0.001$ (Greenhouse-Geisser corrections for lack of sphericity were applied), a trend analysis indicated that the linear trend was significant, $\mathrm{p}<0.001$, as was the quadratic term, $\mathrm{p}<0.002$; for Digit span forward, $\mathrm{F}(9,126)=$ $4.8, \mathrm{p}<0.001$, a trend analysis indicated that the linear trend was significant, $\mathrm{p}<0.006$, as was the cubic term, $\mathrm{p}<0.05$; for Corsi block tapping forward, $\mathrm{F}(9,99)=2.0$, $\mathrm{p}<0.05$, a trend analysis indicated that the linear trend was significant, $\mathrm{p}<0.01$, and there was a trend for the quadratic term, $\mathrm{p}<0.09$; for Corsi block tapping backwards, $\mathrm{F}(9,81)=3.8, \mathrm{p}<0.001$, a trend analysis indicated that the linear trend was significant, $\mathrm{p}<0.001$. For Digit span backwards, there was a marginally significant effect for changes in performance across training sessions: $F(9$, $126)=1.8, \mathrm{p}<0.07$, a trend analysis indicated that the linear trend was approaching significance, $\mathrm{p}<0.06$, as was the cubic term, $\mathrm{p}<0.08$. To further illustrate these training trends, the two training weeks were examined separately. Paired t tests comparing the first and last day of each training week revealed significant performance increases in the first week of training (1st to 5th day) for K-ABC Icons $(t(19)=-5.0, p<0.001)$, and a trend towards significance for Digit span backwards $(\mathrm{t}(19)=-1.9, \mathrm{p}<$ $0.07)$ and Corsi block tapping forward $(\mathrm{t}(18)=-1.8, \mathrm{p}<$ 0.08 ). In contrast, in the second week of training (6th to 10th day) performance increased significantly for Corsi block tapping backwards $(\mathrm{t}(17)=-2.7, \mathrm{p}<0.02)$ and Digit span backwards $(\mathrm{t}(17)=-2.1, \mathrm{p}<0.05)$. None of the other comparisons were significant. Analyses of effect sizes revealed similar results: in the first week of training, medium to large training effects were obtained for KABC Icons $\left(\mathrm{d}^{\prime}=0.79\right)$ and Corsi block tapping forward $\left(d^{\prime}=0.44\right)$, whereas in the second week medium to large training effects were found for Corsi block tapping backwards $\left(\mathrm{d}^{\prime}=0.75\right)$ and Digit span backwards $\left(\mathrm{d}^{\prime}=0.40\right)$. All other effect sizes were small.

\section{Exploring Differential Training Effects}

Next, to determine who profited most, the training group was divided via baseline median split into approx-

\footnotetext{
2 Considering the available normative data from the MWT-A, mean verbal cognitive abilities of both low- and high-capacity individuals fell within the normal to high functioning range of their population.
}

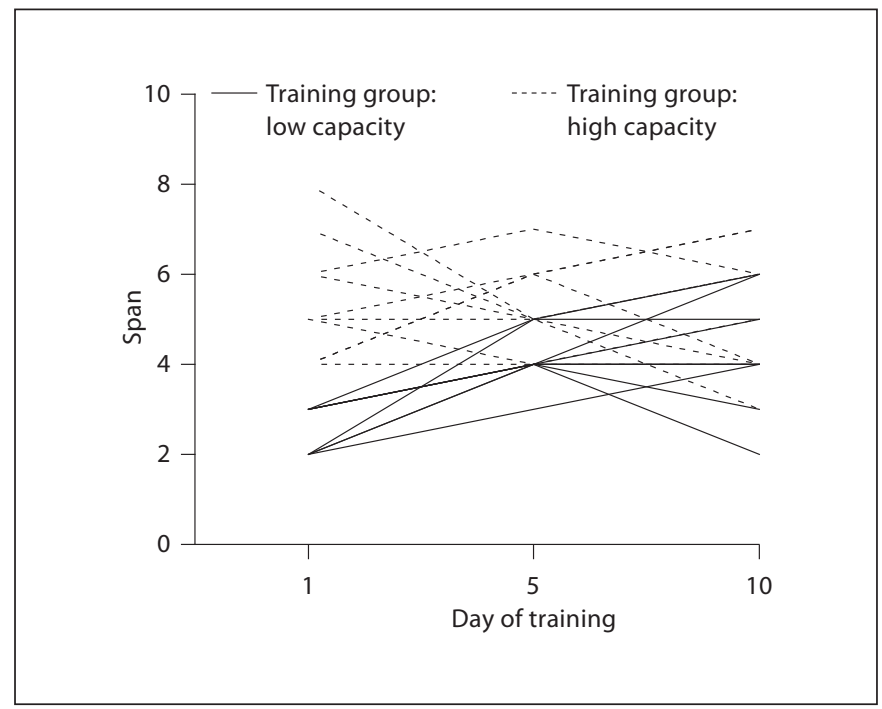

Fig. 2. Performance trajectories in Corsi block tapping forward across training (training days 1, 5 and 10) of training group individuals with low vs. high baseline WM capacity.

imately equally sized groups of high- and low-capacity individuals for each of the trained WM tasks. ${ }^{2}$ In a first step, within each subgroup, pre- and post-training comparisons revealed significant training gains in all trained tasks for low-capacity individuals (Digit span forward: $\mathrm{t}(7)=-3.4, \mathrm{p}<0.01$; Digit span backwards: $\mathrm{t}(12)=-3.9$, $\mathrm{p}<0.01$; Corsi block forward: $\mathrm{t}(9)=-3.8, \mathrm{p}<0.01$; Corsi block backwards: $\mathrm{t}(12)=-8.8, \mathrm{p}<0.001$; K-ABC Icons: $\mathrm{t}(9)=-5.6, \mathrm{p}<0.001$, respectively), whereas there were no significant training gains for high-capacity individuals. $^{3}$ Figure 2 exemplifies the longitudinal performance trajectories in the Corsi block forward task during training for all participants of the training group, separating low- and high-capacity subgroups. Low-capacity individuals generally showed increases in performance across the training period, whereas high-capacity individuals mostly demonstrated either no major changes or decreases in performance. The patterns of trajectories for the low- and high-capacity individuals were similar for all other training tasks.

\footnotetext{
3 To control for task-specific effects, analyses were repeated with an overall WM sum score for each individual (sum of scores on all WM tasks on training day 1). The pattern of results was supported: participants with a sum score above the median did not show any significant training gains, whereas for participants with a sum score below the median significant training gains were found with the exception of the Corsi block tapping task forward.
} 


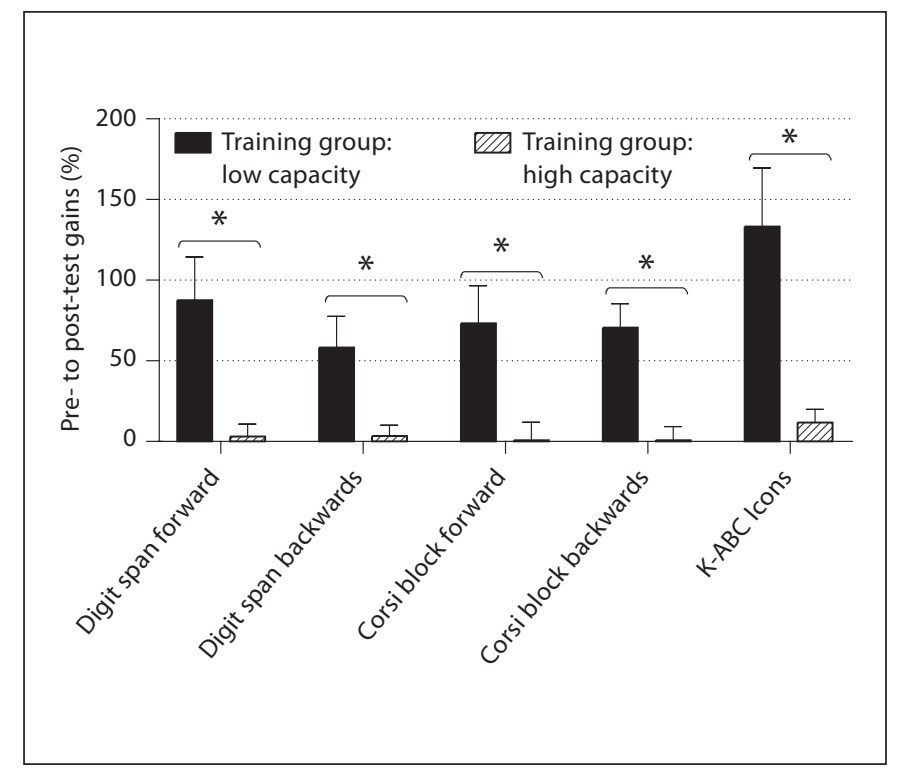

Fig. 3. Comparisons between high- and low-capacity training group individuals for mean training gains in percent for each training task separately. Error bars indicate standard errors. Significant differences of training gains between high- and low-capacity groups are indicated: ${ }^{*} \mathrm{p}<0.05$.

In a second step, high-capacity individuals' training gains were directly compared to those of low-capacity individuals. Significantly larger training gains for the low-capacity group were found for all training tasks: all $\mathrm{p}<0.02$. Furthermore, in a third step, trainings gains were compared between the low-capacity training group and the control group. Confirming the previously found pattern, trainings gains were significantly larger in the low-capacity training group than in the control group for all training tasks as well: all $\mathrm{p}<0.05$ (see fig. 3 and table 2 for further details).

The relationship between baseline WM capacity and training gains was further supported by the following findings. Correlation analyses revealed significant large negative correlations between baseline WM capacity and training gains for all trainings tasks (Digit span forward: $r=-0.66, p<0.002$; Digit span backwards: $r=-0.59$, $\mathrm{p}<0.006$; Corsi block tapping forward: $\mathrm{r}=-0.65$, $\mathrm{p}<0.002$; Corsi block tapping backwards: $\mathrm{r}=-0.89$, $\mathrm{p}<0.001$; K-ABC: $\mathrm{r}=-0.80, \mathrm{p}<0.001) .{ }^{4}$

4 We thank an anonymous reviewer for suggesting this additional analysis.

\section{Exploring Transfer Effects to Nontrained Executive}

Functions Tasks

Paired $t$ tests comparing pre- and post-training performance in non-trained executive functions tasks revealed that there was a trend for improvement in Stroop interference scores in the training group $(\mathrm{t}(17)=2.0$, $p<0.06, d^{\prime}=0.3$ ), whereas for the control group no significant differences were found $\left(\mathrm{t}(15)=1.0, \mathrm{p}<0.3, \mathrm{~d}^{\prime}=\right.$ $0.2)$. For Raven sum scores, the control group improved significantly from pre- to postassessment $(\mathrm{t}(15)=-2.8$, $\mathrm{p}<0.01, \mathrm{~d}^{\prime}=0.4$ ), whereas the difference between preand post scores was not significant for the training group $\left(\mathrm{t}(19)=-1.5, \mathrm{p}<0.15, \mathrm{~d}^{\prime}=0.2\right)$. Importantly, independent $t$ tests revealed no significant differences between groups in percent gains in either Stroop interference scores (training group: $\mathrm{M}=-9.6 \%, \mathrm{SD}=30.9$, control group: $\mathrm{M}=-2.7 \%, \mathrm{SD}=30.6 ; \mathrm{t}(32)=-0.65, \mathrm{p}>0.1)$ or Raven scores (training group: $\mathrm{M}=5.2 \%, \mathrm{SD}=11.5$, control group: $\mathrm{M}=11.5 \%, \mathrm{SD}=16.9 ; \mathrm{t}(34)=-1.32$, $\mathrm{p}>0.1)$.

\section{Discussion}

The present research is the first study on WM plasticity in older adults that are well within the old-old age range. Results provide initial evidence that (1) training gains in WM capacity are possible even in old-old age, and (2) when applying ten training sessions only. More detailed analyses of the training progress indicated changes in performance over the ten training sessions in almost all tasks that mostly reflected linear ascending trends, with some accelerated components. Moreover, the study is the first to demonstrate that WM plasticity in old-old age is preserved in both WM modalities as verbal and visuospatial training effects were revealed. (3) With respect to our third research question, results showed that within the training group, individuals with low WM capacity were driving the training effects. While those findings demonstrate potential for behavioral plasticity in old-old age, the present study also suggests some limits. Specifically, gains in trained tasks were higher in the training group than in a passive control group for only some of the tasks. This may have been caused by smaller training gains of participants with high WM capacity (see below for the issue of differential subgroup effects). Furthermore, there was no clear indication of transfer effects of the WM training to nontrained executive functions tests. 
Table 2. Percent gains in trained tasks for individuals of the training group with low vs. high working memory capacity and individuals of the control group

\begin{tabular}{|c|c|c|c|c|c|c|c|c|c|c|}
\hline & \multicolumn{4}{|c|}{ Training group, mean $\pm \mathrm{SD}$} & \multicolumn{2}{|c|}{$\begin{array}{l}\text { Control group } \\
\text { mean } \pm \text { SD }\end{array}$} & \multicolumn{2}{|c|}{$\begin{array}{l}\text { Low vs. high } \\
\text { WM capacity }\end{array}$} & \multicolumn{2}{|c|}{$\begin{array}{l}\text { Low WM capac- } \\
\text { ity vs. control }\end{array}$} \\
\hline & \multicolumn{2}{|c|}{ low WM capacity } & \multicolumn{2}{|c|}{ high WM capacity } & \multirow[b]{2}{*}{$\%$} & \multirow[b]{2}{*}{$\mathrm{n}$} & \multirow[b]{2}{*}{$\mathrm{p}$} & \multirow[b]{2}{*}{$d^{\prime}$} & \multirow[b]{2}{*}{$\mathrm{p}$} & \multirow[b]{2}{*}{$d^{\prime}$} \\
\hline & $\%$ & $\mathrm{n}$ & $\%$ & $\mathrm{n}$ & & & & & & \\
\hline Digit span forward & $87.5 \pm 76.0$ & 8 & $3.1 \pm 26.5$ & 12 & $-9.0 \pm 32.7$ & 16 & 0.02 & 1.6 & 0.008 & 1.8 \\
\hline Digit span backwards & $58.3 \pm 69.2$ & 13 & $3.5 \pm 17.6$ & 7 & $16.6 \pm 36.9$ & 16 & 0.02 & 0.9 & 0.05 & 0.8 \\
\hline Corsi forward & $73.3 \pm 73.4$ & 10 & $0.7 \pm 35.4$ & 10 & $1.4 \pm 25.3$ & 16 & 0.02 & 1.2 & 0.01 & 1.4 \\
\hline Corsi backwards & $70.5 \pm 53.8$ & 13 & $0.7 \pm 22.3$ & 7 & $18.4 \pm 35.0$ & 16 & 0.004 & 1.5 & 0.004 & 1.1 \\
\hline K-ABC Icons & $133.3 \pm 114.4$ & 10 & $11.8 \pm 25.6$ & 10 & $48.8 \pm 54.6$ & 16 & 0.008 & 1.4 & 0.05 & 1.0 \\
\hline
\end{tabular}

Mean values and SD are indicated for each subgroup. Percent gains in each task were compared between groups using independent $\mathrm{t}$ tests. Significance levels (p) and effect sizes ( $\left.\mathrm{d}^{\prime}\right)$ for all comparisons are indicated.

Overall, the present results replicate and extend the previous literature in several ways. First, training gains of the current study were comparable in size to those observed by Buschkuehl et al. [16] in a sample on the edge to old-old age (mean age 80 years). Gains on the three WM tasks used by Buschkuehl and colleagues were 44, 62 and $15 \%$, respectively, while in the present study the training gains ranged from 37 to $72 \%$. Second, when looking at the low WM capacity individuals who were mainly driving the training effects, training gains in the present study were even larger, ranging from 58 to $133 \%$. Thus, the WM training adopted in this study, in spite of its overall much shorter duration (10 sessions) as compared to the training regime by Buschkuehl et al. [16] (23 sessions with WM tasks trained consecutively), seems to be very effective in improving WM performance in old-old adults with low WM capacity. In fact, in comparison to the control group, significant training effects were found in all tasks for this subgroup. However, since no significant training effects were found for the high-capacity individuals, the present training regime does not seem to be suitable for high-functioning old-old adults.

Third, differential training effects were observed with respect to process requirements of the trained tasks. First of all, significant group differences in training gains (indicating substantial training effects) were only found for some tasks: Digit span forward and Corsi block tapping forward. Since these two tasks mostly require storage of information, this may suggest that the training regime applied may have been more effective for improving storage rather than processing components of WM. More- over, as indicated by the follow-up analyses to the longitudinal trends observed in the repeated measures ANOVAs, in the first training week, training gains were mainly larger for tasks focusing on storage of information (Corsi block tapping forward, K-ABC Icons) than for tasks also involving processing components (Corsi block tapping backwards, Digit span backwards). These findings indicate that more training time may have been necessary to improve processing components of WM in oldold adults, possibly because the processing components of WM are more affected in old age than the storage components [11]. Alternatively, it is possible that the processing component of WM may not be modifiable in old-old age at all. Future research will have to address this issue in further detail.

As indicated above, differential patterns also emerged with respect to individual differences in ability levels at the beginning of training. Conceptually, the finding of large training gains in the low-capacity individuals and virtually none in the high-capacity individuals appears to be consistent with the disuse hypothesis [21, 22]. The disuse hypothesis assumes that cognitive decline in old age may be due to suboptimal use of cognitive resources. Frequent use of cognitive resources is thought to help maintain high performance levels, whereas lack of practice is thought to lead to low performance levels. In the current study, the individuals with low baseline WM capacity may have lacked frequent practice of WM tasks. When challenged in an adaptive training regime they seemed to be able to reactivate some of their potential. Participants with high baseline WM capacity may have maintained their ability through frequent use of WM 
processes and therefore might not have profited as much from the applied WM training that focused on practice of these processes. However, current findings are in contrast to previous data showing greater training gains for individuals with higher baseline capacity [18-20]. These divergent findings could be explained by different methods to define high and low capacity. Previous studies often defined capacity not by the ability the training focused on, but by general measures of cognitive status such as the MMST or intelligence tests [18-20]. In contrast, in the present study, capacity was defined by baseline performance in the trained tasks, thus using a more direct approach to examine the influence of capacity on training gains. In addition, in some of the previous studies, low-capacity individuals were considered as being at risk for development of dementia [19, 20], thus limiting their ability to profit from training, whereas in the present study only participants were included who passed a screening test for preclinical and clinical dementia. Furthermore, the training regimes applied in the previous studies focused on teaching and practicing rather complex mnemonic strategies $[19,20]$ or depended on the participants' ability to self-initiate the use of encoding strategies [18], thus drawing on a great amount of cognitive resources for successful implementation of the respective training mechanisms. In contrast, the focus of the WM training applied in the current study was adaptive practice of storing and processing of information. Moreover, the WM training in this study may have been particularly tailored for low-capacity individuals by using paper-pencil training tasks with more liberal reaction time limits as compared to computerized trainings previously used, thus enhancing motivation and engagement with the training tasks. This view is supported by the high compliance to the training.

Despite improvements in the trained tasks, the current study did not provide evidence for (far) transfer to executive control tests. At first sight, this is not in line with previous findings of transfer effects after WM training in old age [14-16]. However, the previously reported transfer effects were restricted with regard to cognitive domain, type of stored information, and involved processes: they were only found in tasks rather similar in these criteria to the trained ones, thus only demonstrating near instead of far transfer. The executive control tests used in the present study to evaluate transfer may have differed too much in these regards from the training tasks to find any transfer. Processes that are thought to be shared between executive functions and WM tasks are those that guide processing of stored information in WM (i.e. the central ex- ecutive component of WM) [4]. Since in the present study, training gains were mainly observed in tasks focusing on the storage of information and were just starting to evolve in the second training week for training tasks also requiring processing of the stored information, a longer training period may have been needed to observe transfer to executive functions tests. As Dahlin et al. [14] - who also did not find transfer to executive functions tests in young and young-old adults with an even longer training (15 sessions) - pointed out a high level of skill may be required for the occurrence of such far transfer. Consequently, old-old adults who have clear WM deficits in comparison to younger adults may need particularly long training regimes to transfer the practiced skill to other tasks.

Limitations of the present study include the small sample size that made it difficult to demonstrate statistical significance for smaller effects, possibly leading to non-detection of transfer effects. However, power was sufficient to detect training effects. Dividing the training group via median split into high- and low-capacity individuals led to even smaller groups, therefore the findings of these analyses should be considered to be exploratory. Acknowledging the exploratory nature of the findings, only the general pattern of results was interpreted, not single nonsignificant results. Follow-up studies on the subject should include a greater number of participants to thoroughly test the current exploratory findings despite the challenges in recruiting old-old adults for time-consuming training studies (also note that Dahlin et al. [14] and Buschkuehl et al. [16] had sample sizes similar or even smaller than the present study). Furthermore, to control for the effects of social interactions on training gains which we cannot completely rule out in our study, the engagement of a control group in a placebo training regime would be desirable (again note that Dahlin et al. [14] also used a no-contact control group). Follow-up studies should also include a wider range of tests to evaluate near and far transfer effects to allow for a more fine-grained analysis of the potential and limits of transfer induced by WM training in oldold age and the potential influence of cognitive domain, type of memory content, or involved processes. In addition, the experimental evaluation of different training lengths may be useful for determining the most efficient training duration.

In summary, the findings of the present study demonstrate there is potential for cognitive plasticity in old-old age in low-capacity individuals, induced by a short WM training particularly tailored for this population. Find- 
ings support the notion that regardless of age, practice on highly demanding tasks can improve performance. However, the present findings also point to possible limits of cognitive plasticity in old-old age: transfer of training gains may be only attainable with very long training regimes and only to very similar tasks.

\section{Acknowledgement}

We thank the participants of the study for their time and effort.

\section{Disclosure Statement}

None.

\section{References}

1 Park DC, Lautenschlager G, Hedden T, Davidson NS, Smith AD, Smith, PK: Models of visuospatial and verbal memory across the adult life span. Psychol Aging 2002;17:299300.

2 Verhaeghen P, Marcoen A, Goossens L: Improving memory performance in the aged through mnemonic training: a metaanalytic study. Psychol Aging 1992;7:242-251.

3 Singer T, Lindenberger U, Baltes PB: Plasticity of memory for new learning in very old age: a story of major loss? Psychol Aging 2003;18:306-317

4 Baddeley A: Working memory: looking back and looking forward. Nat Rev Neurosci 2003;4:829-839.

5 Conway ARA, Cowan N, Bunting MF, Therriault DJ, Minkoff SRB: A latent variable analysis of working memory capacity, shortterm memory capacity, processing speed, and general fluid intelligence. Intelligence 2002:30:163-183.

6 Engle RW, Tuholski SW, Laughlin JE, Conway AR: Working memory, short-term memory, and general fluid intelligence: a latent-variable approach. J Exp Psychol Gen 1999;128:309-331.

7 Klingberg T, et al: Computerized training of working memory in children with ADHD - a randomized, controlled trial. J Am Acad Child Adolesc Psychiatry 2005;44:177-186.

8 Holmes J, Gathercole SE, Dunning DL: Adaptive training leads to sustained enhancement of poor working memory in children. Dev Sci 2009;12:F9-F15.

9 Jaeggi SM, Buschkuehl M, Jonides J, Perrig WJ: Improving fluid intelligence with training on working memory. Proc Natl Acad Sci USA 2008; 105:6829-6833.

10 Bopp KL, Verhaeghen P: Aging and verbal memory span: a meta-analysis. J Gerontol B Psychol Sci Soc Sci 2005;60:223-233.

11 Craik FIM: Age-related changes in human memory; in Park DC, Schwarz N (eds): Cognitive Aging: A Primer. Philadelphia, Psychology Press, 2000, pp 75-92.
12 Gilinsky AS, Judd BB: Working memory and bias in reasoning across the adult life span. Psychol Aging 1994;9:356-371.

13 Buchler NG, Hoyer WJ, Cerella J: Rules and more rules: the effects of multiple tasks, extensive training, and aging on task-switching performance. Mem Cognit 2008;36:735748.

14 Dahlin E, Nyberg L, Bäckman L, Neely AS: Plasticity of executive functioning in young and older adults: immediate training gains, transfer, and long-term maintenance. Psychol Aging 2008;23:720-730.

15 Li SC, Schmiedek F, Huxhold O, Röcke C, Smith J, Lindenberger U: Working memory plasticity in old age: practise gain, transfer and maintenance. Psychol Aging 2008;23: 731-742.

16 Buschkuehl $\mathrm{M}$, et al: Impact of working memory training on memory performance in old-old adults. Psychol Aging 2008;23. 743-753

17 Mayr U: Introduction to the special section on cognitive plasticity in the aging mind. Psychol Aging 2008;23:681-683.

18 Bissig D, Lustig C: Who benefits from memory training? Psychol Sci 2007;18:720-726.

19 Baltes MM, Kühl KP, Gutzmann H, Sowarka D: Potential of cognitive plasticity as a diagnostic tool: a cross-validation and extension. Psychol Aging 1995;10:167-172.

20 Yesavage JA, Sheikh JI, Friedman L, Tanke E: Learning mnemonics: roles of aging and subtle cognitive impairment. Psychol Aging 1990;5:133-137.

21 Gatz M, et al: Education and the risk of $\mathrm{Al}$ zheimer's disease: findings from the study of dementia in Swedish Twins. J Gerontol 2001; 56B,P292-P300.
22 Kliegel M, Zimprich D, Rott C: Life-long intellectual activities mediate the predictive effect of early education on cognitive impairment in centenarians: a retrospective study. Aging Ment Health 2004;8:430-437.

23 Kliegel M, Rott C, d'Heureuse V, Becker G, Schönemann P: Demenz im höchsten Alter ist keine Notwendigkeit. Ergebnisse der Heidelberger Hundertjährigen-Studie. Z Gerontopsychol Psychiatr 2001;14:169-180.

24 Rott C, d'Heureuse V, Kliegel M, Schönemann P, Becker G: Die Heidelberger Hundertjährigen-Studie: theoretische und methodische Grundlagen zur sozialwissenschaftlichen Hochaltrigkeitsforschung. Z Gerontol Geriatr 2001;34:356-364.

25 Lehrl S, Merz J, Burkhard G, Fischer S: MWT-A: Mehrfachwahl-Wortschatz-Intelligenztest. Göttingen, Hogrefe, 1991.

26 Klingberg T, Forssberg H, Westerberg H: Training of working memory in children with ADHD. J Clin Exp Neuropsychol 2002; 24:781-791.

27 Tewes U: HAWIE-R. Hamburg-WechslerIntelligenztest für Erwachsene - Revision, ed 2, revised. Göttingen, Hogrefe, 1994.

28 Berch DB, Krikorian R, Huha EM: The Corsi block-tapping task: methodological and theoretical considerations. Brain Cogn 1998; 38:317-338.

29 Melchers P, Preuss U: Kaufman assessment battery for children: K-ABC. Frankfurt am Main, Swets \& Zeitlinger, 1991.

30 Bäumler G: Farbe-Wort-Interferenztest nach J. R. Stroop (FWIT). Göttingen, Hogrefe, 1985.

31 Raven JC, Court JH, Raven J: Coloured Progressive Matrices. London, Lewis, 1976.

32 Hedges LV, Olkin I: Statistical Methods for Metaanalysis. Orlando, Academic Press, 1985.

33 Cohen J: Statistical Power Analysis for the Behavioural Sciences, ed 2. Hillsdale, Lawrence Erlbaum, 1988. 